See discussions, stats, and author profiles for this publication at: https://www.researchgate.net/publication/284076085

\title{
Variational theory of complex rays applied to shell structures: in-plane inertia, quasi-symmetric ray distribution, and orthotropic materials
}

Article in Computational Mechanics · October 2015

DOI: 10.1007/s00466-015-1214-6

\section{CITATIONS}

4 authors:

Alessandro Cattabiani

Technische Universität München

5 PUBLICATIONS 5 CITATIONS

SEE PROFILE

Hervé Riou

Ecole normale supérieure de Cachan

36 PUBLICATIONS 260 CITATIONS

SEE PROFILE
109

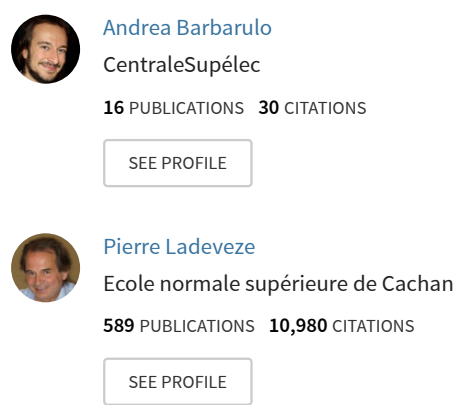

Some of the authors of this publication are also working on these related projects:

Project Modeling damage behavior of interleaved laminates View project

Project Numerical Methods for medium frequency vibro-acoustics in Laboratory LMT ENS Paris-Saclay View project 


\title{
Variational Theory of Complex Rays applied to shell structures: in-plane inertia, quasi-symmetric ray distribution, and orthotropic materials
}

\author{
Alessandro Cattabiani · Andrea Barbarulo · Hervé Riou · Pierre Ladevèze
}

Received: date / Accepted: date

\begin{abstract}
Recently, interest of aerospace and automotive industries on medium-frequency vibrational behavior of composite shell structures has grown due to their high specific stiffness and fatigue resistance. Conventional methods such as the Finite Element Method (FEM) and the Statistical Energy Analysis(SEA) are not suitable for the medium-frequency bandwidth. Conversely, the Variational Theory of Complex Rays (VTCR) is taking place as an ad-hoc technique to tackle such frequency band. It is a Trefftz method based on a weak variational formulation. Equilibrium equations are met using exact solutions as shape functions. The variational problem imposes boundary conditions in weak form. The present paper extends VTCR to orthotropic shell structures. Moreover, several new enhancements are introduced. Now, we use a quasi-symmetric ray distribution which can greatly reduce computational costs, and addresses in-plane inertia which was neglected in previous works. Some relevant numerical examples are presented to show the strategy and re-
\end{abstract}

The authors gratefully acknowledge the "Centre National d'Études Spatiales (CNES)" and "Airbus Defense and Space".

\footnotetext{
A. Cattabiani

LMT-Cachan (ENS Cachan/CNRS/Paris-Saclay-University), 61 avenue du Président Wilson, F-94230 Cachan, France

Tel.: +33 (0) 695979893

Fax: $+33(0) 147402785$

E-mail: cattabiani@1mt.ens-cachan.fr

A. Barbarulo

Laboratoire MSSMat (Centrale-Supélec / CNRS UMR8579) Grande Voie des Vignes, Cedex, Chatenay-Malabry, 92290, France

H. Riou

LMT-Cachan (ENS Cachan/CNRS/Paris-Saclay-University), 61 avenue du Président Wilson, F-94230 Cachan, France

P. Ladevèze

LMT-Cachan (ENS Cachan/CNRS/Paris-Saclay-University), 61 avenue du Président Wilson, F-94230 Cachan, France
}

sults are compared with a FEM reference to study performances.

Keywords VTCR · mid-frequency · orthotropic materials . shells

\section{Introduction}

In recent years, aerospace and automotive industries have greatly increased use of composite shell structures due to their high specific stiffness and fatigue resistance. This has fostered a focused interest towards virtual testing of their vibrational behavior over a large frequency band. Since the qualitative behavior of a structure drastically varies with frequency, there are many optimized methods in literature to study a vibrational problem over a specific part of the frequency range. The Modal Overlap Factor (MOF) [28] identifies three zones: low, mid and high frequency range.

The low-frequency range can be effectively tackled by deterministic methods such as the Finite Element Method (FEM) [18] or the Boundary Element Method (BEM) [15]. These methods are limited to low-frequency since at midand high-frequency the phenomena variation length is very small if compared to characteristic dimensions of the structure [9]. This produces numerical instability known as pollution effect. In order to counteract it, Ihlenburg in [20] proved that the parameter $k^{3} h^{2}$ must be kept constant where $k$ is the wave number and $h$ is the element size. For this reason, in medium-frequency range computational costs become prohibitive. Yet, many approaches were presented to overcome these difficulties such as the isogeometric analysis (IGA) [16, 34], the Galerkin Least-Squares FEM (GLS-FEM) [13], the Finite Element Tearing and Interconnecting (FETI) [11], and the variational multiscale FEM [17].

The high-frequency range can be addressed by the Statistical Energy Analysis (SEA) [28, 29] and its developments 
such as the Wave Intensity Analysis (WIA)[26] or Beam Tracing (BT) [40]. These techniques study the energy flux among subdomains neglecting almost entirely spatial quantities. These approaches are based on assumptions that the wave field is reverberant and that the modal density is high enough. These hypothesis are usually assured only in the high-frequency range. Nevertheless, a lot of work has been done to extend such theories to medium-frequency range [11, 27, 37].

A class of methods has been specifically developed for the medium-frequency range such as the partition of unity method[36, 1], the ultra-weak variational method [4, 19], the least-squares method [30], the asymptotic scaled modal analysis [6], the energy operator eigenmodes [35], the discontinuous enrichment method [12], the element-free Galerkin method [3], the wave boundary element method [31] or the wave-based method [10, 14, 7, 8]. The present work further develops one of them: the Variational Theory of Complex Rays (VTCR) [24]. This method approximates the vibrational problem solution as a sum of shape functions that identically satisfy equilibrium equations and addresses boundary conditions in weak form. This approach allows a priori independent approximations among subdomains. Thus, different (in number and type) shape functions can be chosen for each subdomain providing great flexibility. Since its first introduction in [24] where it were applied on plate theory, VTCR has been implemented in a lot of different fields such as transient dynamics [5], 3D acoustic [22], and on a wide frequency band [2]. In particular, Riou in [32] applied VTCR to general shell theory neglecting in-plane inertia and Kovalevsky in [23] extended the method to orthotropic plates. This work merges and further develops such studies in three ways:

1. orthotropic materials are included in the VTCR formulation for the general shell theory,

2. in-plane inertia is addressed adding two type of propagative waves that carry in-plane stresses and displacements,

3. a quasi-symmetric ray distribution is introduced to drastically reduce computational costs.

After the theory is introduced, some relevant numerical examples are presented to validate the strategy. The first one focuses on a comparison among VTCR with and without inplane inertia and a FEM reference. Performances and convergence ratios are studied over a frequency band. The second example involves orthotropic materials: the VTCR solution is compared with a FEM one at a fixed frequency and performances are investigated.

\section{General shell theory}

In this Section are examined the equilibrium and boundary equations of the general kirchhoff-Love theory for orthotropic shells. The present development is akin to the one provided in [39] and [38].

\subsection{Equilibrium equations}

The general reference example is presented in 1 The focus is on a generic subdomain $\Omega_{i}$ of the frame structure in Figure 1. For the sake of clarity various boundary, corner, coupling, and surface conditions are split in Figure 2 The $\partial_{\square} \Omega_{i}$ symbol refers to a generic boundary of $\Omega_{i}$ where condition $\square$ is applied. In the particular case of a boundary shared among subdomains, $\Gamma$ is used instead. In the same way, for conditions applied on corners, a symbol $\partial \partial_{\square} \Omega_{i}$ is used. The generic corner shared among subdomains is indicated with $\mathscr{C}$. The over-line symbol $\square$ indicates that a quantity $\square$ is known (i.e. a value of a boundary constraint). $\hat{\mathbf{n}}_{i}$ indicates a normal unit vector of a boundary directed outward $\Omega_{i}$. A generic subdomain $\Omega_{i}$ is subject to loads, displacements constraints, and continuity conditions along boundaries (Figure 2a) and on corners (Figure 2b) as well as a distributed load per unit surface $\overline{\mathbf{g}}_{i}$ (Figure 2d). Without loss of generality, a displacement constraint $\overline{\mathbf{u}}_{i}=\left[\overline{\mathbf{v}}_{i}^{\prime}, \bar{w}_{i}\right]^{\prime}$ along $\partial_{\mathbf{u}_{i}} \Omega_{i}$ can be divided in in-plane $\overline{\mathbf{v}}_{i}$ and out-of-plane $\bar{w}_{i}$ components 1 In the same way, a load per unit length $\overline{\mathbf{p}}_{i}=\left[\overline{\mathbf{b}}_{i}^{\prime}, \bar{q}_{i}\right]^{\prime}$ along $\partial_{\overline{\mathbf{p}}_{i}} \Omega_{i}$ can be divided in in-plane $\overline{\mathbf{b}}_{i}$ and out-of-plane $\bar{q}_{i}$ components. A rotation condition $\bar{w}_{i, \hat{\mathbf{n}}_{i}}$ is im-

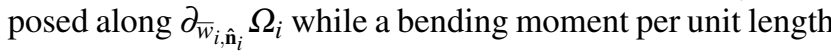
$\bar{m}_{i}$ is applied along $\partial_{\bar{m}_{i}} \Omega_{i}$. Corners are subject to out-ofplane displacements constraints $\bar{w}_{\mathscr{C} i}$ on $\partial \partial_{\bar{w}_{\mathscr{C}} i} \Omega_{i}$ and point forces $\bar{q}_{\mathscr{C} i}$ on $\partial \partial_{\bar{q}_{\mathscr{C} i}} \Omega_{i}$. Coupling conditions are applied on $\mathscr{C}$ and along $\Gamma$, in order to ensure stresses and displacements continuity among subdomains (Figure 2c).

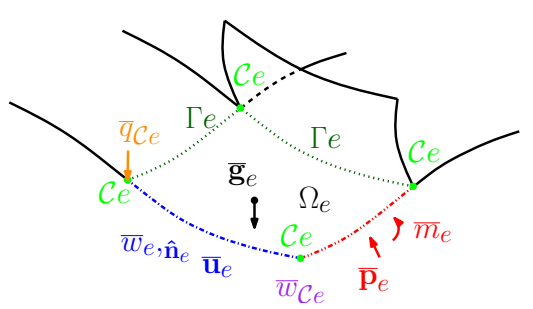

Fig. 1: Generic frame structure described in Section 2.1

All quantities of interest are defined in the complex domain. Each one is considered multiplied by $e^{j \omega t}$ where $j=$

${ }^{1} \square^{\prime}$ is the transpose operator. 


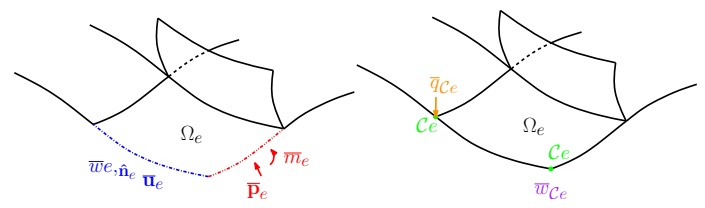

(a) boundary conditions.

(b) corner conditions.



(c) coupling conditions.

(d) surface conditions.

Fig. 2: Boundary, corner, coupling and surface constraints of the generic frame structure of Figure 1 subdivided and hightlighted.

$\sqrt{-1}$ is the imaginary unit, $\omega=2 \pi f$ is the angular frequency and $t$ is the time.

Curvilinear coordinates $\left\{\hat{\alpha}_{i}, \hat{\beta}_{i}, \hat{\mathbf{z}}_{i}\right\}$ define shell geometry. $\left\{\hat{\alpha}_{i}, \hat{\beta}_{i}\right\}$ are unit vectors orthogonal each other and tangent to shell middle surface in every point. $\hat{\mathbf{z}}_{i}$ is the outof-plane coordinate and is determined in each surface point by $\hat{\mathbf{z}}_{i}=\hat{\alpha}_{i} \times \hat{\beta}_{i}$ where $\times$ is the cross product. The generic surface is described by the vector field

$\mathbf{r}_{i}=\mathbf{r}_{i}(\alpha, \beta)$

where $\mathbf{r}_{i}$ is the generic $3 \mathrm{D}$ position vector of subdomain $\Omega_{i}$. Lamé surface parameters $\left\{L_{\alpha i}, L_{\beta i}\right\}$ and curvature radii $\left\{R_{\alpha i}, R_{\beta i}\right\}$ are

$L_{\alpha i}=\sqrt{\mathbf{r}_{i, \alpha} \cdot \mathbf{r}_{i}, \alpha}$,

$L_{\beta i}=\sqrt{\mathbf{r}_{i, \beta} \cdot \mathbf{r}_{i, \beta}}$,

$R_{\alpha i}=\frac{L_{\alpha i}^{2}}{\hat{\mathbf{z}}_{i} \cdot \mathbf{r}_{i}, \alpha \alpha}$

$R_{\beta i}=\frac{L_{\beta i}^{2}}{\hat{\mathbf{z}}_{i} \cdot \mathbf{r}_{i}, \beta \beta}$,

where commas indicate directional derivatives. These definitions will be useful in the following Sections. Displacement field can be restricted to (Kirchhoff's kinematics assumptions)

$\mathbf{u}_{i}^{z}=\mathbf{u}_{i}-z_{i} \phi_{i}$,

$\phi_{i}=\nabla w_{i}-\mathrm{R}_{i} \cdot \mathbf{v}_{i}$

$\mathrm{R}_{i}=\left[\begin{array}{cc}\frac{1}{R_{\alpha i}} & 0 \\ 0 & \frac{1}{R_{\beta i}}\end{array}\right]$, where $\mathbf{u}_{i}^{z}$ is the displacement thorough shell thickness, $\mathbf{u}_{i}$, $\mathbf{v}_{i}$ and $w_{i}$ are respectively total, in-plane, and out-of-plane displacements of the middle surface, and $\mathrm{R}_{i}$ is the curvature matrix. The solution is researched in $\mathscr{D}_{i}=\left\{\mathbf{u}_{i}, \mathrm{~N}_{i}, \mathrm{M}_{i}\right\} . \mathbf{u}_{i}$ is a finite energy displacement and $\mathrm{N}_{i}$ and $\mathrm{M}_{i}$ are finite energy generalized stress tensors. $\mathscr{D}_{i}$ satisfies the in-plane equilibrium equation

$\nabla \cdot \mathrm{N}_{i}-\mathrm{R}_{i}\left(\nabla \cdot \mathrm{M}_{i}\right)+\overline{\mathbf{g}}_{\alpha \beta i}+\rho_{i} h_{i} \omega^{2} \mathbf{v}_{i}=0$ over $\Omega_{i}$,

and the out-of-plane equilibrium equation

$\nabla \cdot\left(\nabla \cdot \mathrm{M}_{i}\right)+\mathrm{R}_{i}: \mathrm{N}_{i}+\bar{g}_{z i}+\rho_{i} h_{i} \omega^{2} w_{i}=0$ over $\Omega_{i}$,

where distributed loads $\overline{\mathbf{g}}_{i}$ are divided in in-plane and outof-plane components in this way

$\overline{\mathbf{g}}_{i}=\left[\bar{g}_{\alpha i}, \bar{g}_{\beta i}, \bar{g}_{z i}\right]^{\prime}$,

$\overline{\mathbf{g}}_{\alpha \beta i}=\left[\bar{g}_{\alpha i}, \bar{g}_{\beta i}\right]^{\prime}$.

Matrices $\mathrm{N}_{i}$ and $\mathrm{M}_{i}$ are stresses and stresses moment resultants along thickness respectively. Constitutive relations, also called stress-strain relations, are for orthotropic materials [39]

$\mathrm{M}_{i}=\mathrm{D}_{i}:\left(\nabla \nabla w_{i}+\mathrm{R}_{i} \mathrm{E}_{i}+\mathrm{V}_{\text {icorr }}\right)$,

$\mathrm{V}_{\text {icorr }}=\mathrm{R}_{i}\left[\begin{array}{cc}0 & e_{i(1,2)} \\ e_{i(2,1)} & 0\end{array}\right]$,

$\mathrm{N}_{i}=\mathrm{B}_{i}:\left(\mathrm{E}_{i}-\mathrm{R}_{i} w_{i}\right)$,

$\mathrm{E}_{i}=\left[\nabla \mathbf{v}_{i}\right]_{\text {sym }}=\frac{1}{2}\left(\nabla \mathbf{v}_{i}+\nabla \mathbf{v}_{i}^{\prime}\right)$,

$\mathrm{B}_{i}=\left[\begin{array}{ccc}B_{\alpha i} v_{\alpha \beta i} B_{\beta i} & 0 & \\ v_{\beta \alpha i} B_{\alpha i} & B_{\beta i} & 0 \\ 0 & 0 & B_{G i}\end{array}\right]$,

$B_{\alpha i}=\frac{E_{\alpha i} h_{i}}{1-v_{\alpha \beta i} v_{\beta \alpha i}}$,

$B_{\beta i}=\frac{E_{\beta i} h_{i}}{1-v_{\alpha \beta i} v_{\beta \alpha i}}$,

$B_{G i}=G_{\alpha \beta i} h_{i}$,

$\mathrm{D}_{i}=\left[\begin{array}{ccc}D_{\alpha i} v_{\alpha \beta i} D_{\beta i} & 0 & \\ v_{\beta \alpha i} D_{\alpha i} & D_{\beta i} & 0 \\ 0 & 0 & D_{G i}\end{array}\right]$,

$D_{\alpha i}=\frac{E_{\alpha i} h_{i}^{3}}{12\left(1-v_{\alpha \beta i} v_{\beta \alpha i}\right)}$,

$D_{\beta i}=\frac{E_{2 i} h_{i}^{3}}{12\left(1-v_{\alpha \beta i} v_{\beta \alpha i}\right)}$,

$D_{G i}=\frac{G_{\alpha \beta i} h_{i}}{6}$,

$E_{\alpha i}=E_{\alpha 0 i}\left(1+j \eta_{\alpha i}\right)$,

$E_{\beta i}=E_{\beta 0 i}\left(1+j \eta_{\beta i}\right)$,

$G_{i}=G_{0 i}\left(1+j \eta_{G i}\right)$, 
where $\mathrm{B}_{i}$ and $\mathrm{D}_{i}$ are Hooke's plane stress operators concerning in-plane and out-of-plane stresses respectively, $\rho_{i}$ is the density, $h_{i}$ is the shell thickness, $E_{\alpha 0 i}$ and $E_{\beta 0 i}$ are the Young moduli along directions $\alpha$ and $\beta$ respectively, $\eta_{\alpha i}$ and $\eta_{\beta i}$ are the relative damping coefficients of the Young moduli, $v_{\alpha \beta i}$ and $v_{\beta \alpha i}$ are the Poisson's ratios $(\alpha, \beta)$ and $(\beta, \alpha)$ respectively, $G_{\alpha \beta i}$ is the in-plane shear modulus, $\eta_{G i}$ is its specific damping coefficient, $\square: \square$ is the inner matrix product operator, $[\square]_{\text {sym }}=\frac{1}{2}\left(\square^{\prime}+\square\right)$ is the symmetric part operator, $e_{i(\alpha, \beta)}$ is the component $(\alpha, \beta)$ of the matrix $\mathrm{E}_{i}$, and $\mathrm{N}_{i}$ and $\mathrm{M}_{i}$ are the stress and stress moment resultants tensors respectively. The sub-space of $\mathscr{D}_{i}$ associated with homogenized conditions $\left(\overline{\mathbf{g}}_{i}=0\right)$ is denoted as $\mathscr{D}_{0 i}=\left\{\delta \mathbf{u}_{i}, \delta \mathrm{N}_{i}, \delta \mathrm{M}_{i}\right\}$. This definition will be useful in the next Sections.

\subsection{Boundary conditions}

In order to present a well-posed problem, three conditions must be imposed along each boundary and one on each corner. Boundary and corner conditions presented in Figure 1 can be classified in this way:

1. an in-plane condition, either a displacement constraint or a load per unit length $\left(\overline{\mathbf{v}}_{i}\right.$ or $\left.\overline{\mathbf{b}}_{i}\right)$,

2. an out-of-plane condition, either a displacement constraint or a load per unit length $\left(\bar{w}_{i}\right.$ or $\left.\bar{q}_{i}\right)$,

3. either a rotation or a bending moment per unit length $\left(\bar{w}_{i, \hat{\mathbf{n}}_{i}}\right.$ or $\left.\bar{m}_{i}\right)$

4. an out-of-plane condition, on corners either a displacement constraint or a point load $\left(\bar{w}_{\mathscr{C} i}\right.$ or $\left.\bar{q}_{\mathscr{C} i}\right)$.

Boundary conditions in the most general case are

$$
\begin{aligned}
& \mathbf{v}_{i}=\left\{\begin{array}{cc}
\overline{\mathbf{v}}_{i} & \exists \overline{\mathbf{v}}_{i} \\
\mathbf{v}_{j_{\Gamma}} & \nexists \overline{\mathbf{v}}_{i}
\end{array},\right. \\
& w_{i}=\left\{\begin{array}{cl}
\bar{w}_{i} & \exists \bar{w}_{i} \\
w_{j_{\Gamma}} & \nexists \bar{w}_{i}
\end{array},\right. \\
& w_{i, \hat{\mathbf{n}}_{i}}=\left(\nabla w_{i}\right) \hat{\mathbf{n}}_{i}=\left\{\begin{array}{cc}
\bar{w}_{i, \hat{\mathbf{n}}_{i}} & \exists \bar{w}_{i, \hat{\mathbf{n}}_{i}} \\
w_{j_{\Gamma}, \hat{\mathbf{n}}_{j_{\Gamma}}} & \nexists \bar{w}_{i, \hat{\mathbf{n}}_{i}}
\end{array},\right. \\
& \mathbf{b}_{i}=\mathrm{N}_{i} \hat{\mathbf{n}}_{i}=\overline{\mathbf{b}}_{i}-\sum_{j_{\Gamma}=1}^{n_{\Gamma}} \mathbf{b}_{j_{\Gamma}}, \\
& q_{i}=\left(\nabla \cdot \mathrm{M}_{i}\right) \hat{\mathbf{n}}_{i}+\nabla\left(\hat{\mathbf{t}}_{i}^{\prime} \mathrm{M}_{i} \hat{\mathbf{n}}_{i}\right) \cdot \hat{\mathbf{t}}_{i} \\
& =\left(\nabla \cdot \mathrm{M}_{i}\right) \hat{\mathbf{n}}_{i}+\left(\hat{\mathbf{t}}_{i}^{\prime} \mathrm{M}_{i} \hat{\mathbf{n}}_{i}\right)_{, \hat{\mathbf{t}}_{i}}=\bar{q}_{i}-\sum_{j_{\Gamma}=1}^{n_{\Gamma}} q_{j_{\Gamma}}, \\
& m_{i}=\hat{\mathbf{n}}_{i}^{\prime} \mathrm{M}_{i} \hat{\mathbf{n}}_{i}=\bar{m}_{i}-\sum_{j_{\Gamma}=1}^{n_{\Gamma}} m_{j_{\Gamma}}
\end{aligned}
$$

where $\hat{\mathbf{t}}_{i}$ is the tangent unit vector, $j_{\Gamma}$ is the index relative to the other subdomains sharing with $\Omega_{i}$ the boundary $\Gamma$,

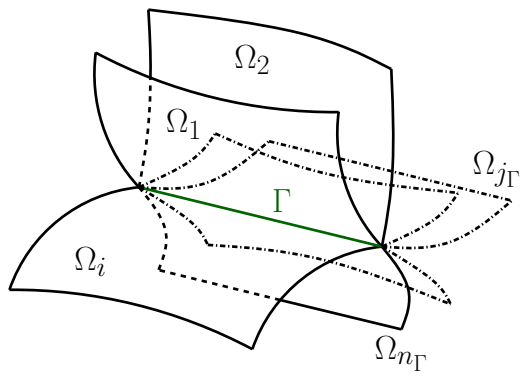

Fig. 3: $n_{\Gamma}+1$ subdomains ( $\Omega_{i}$ included) sharing the same boundary $\Gamma$.

and $n_{\Gamma}$ is their total number ( $\Omega_{i}$ excluded) as is shown in Figure 3.

Corner conditions in the most general case are

$w_{\mathscr{C} i}=\left\{\begin{array}{cc}\bar{w}_{\mathscr{C} i} & \exists \bar{w}_{\mathscr{C} i} \\ w_{\mathscr{C}} j_{\mathscr{C}} & \nexists \bar{w}_{\mathscr{C} i}\end{array}\right.$

$q_{\mathscr{C} i}=\hat{\mathbf{t}}_{1 i}^{\prime} \mathrm{M}_{i} \hat{\mathbf{n}}_{1 i}+\hat{\mathbf{t}}_{2 i}^{\prime} \mathrm{M}_{i} \hat{\mathbf{n}}_{2 i}=\bar{q}_{\mathscr{C} i}-\sum_{j_{\mathscr{C}}=1}^{n_{\mathscr{C}}} q_{j_{\mathscr{C}}}$,

where $\hat{\mathbf{n}}_{1 i}$ and $\hat{\mathbf{n}}_{2 i}$ are outward normal unit vectors of the two boundaries of $\Omega_{i}$ sharing the corner $\mathscr{C} . \hat{\mathbf{t}}_{1 i}$ and $\hat{\mathbf{t}}_{2 i}$ are their respective tangent unit vectors directed towards the corner. As for $j_{\Gamma}$, the index $j_{\mathscr{C}}$ is relative to other subdomains sharing with $\Omega_{i}$ the corner $\mathscr{C} . n_{\mathscr{C}}$ is their total number $\left(\Omega_{i}\right.$ excluded) as is shown in Figure 4.

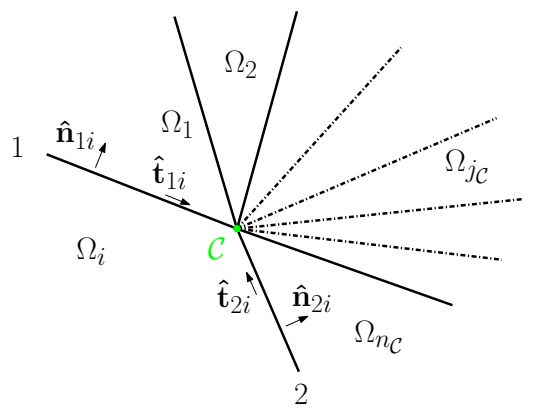

Fig. 4: $n_{\mathscr{C}}+1$ subdomains ( $\Omega_{i}$ included) that share the same corner $\mathscr{C}$.

\section{Shell - VTCR theory}

In this Section a very general version of VTCR for shell structures is illustrated. Since the VTCR is a Trefftz method, the solution is searched in a function set that satisfy equilibrium equations. Boundary and corner residuals are addressed in weak form. The weak variational problem is: 
find the solution set $\mathscr{D}_{s i}=\left\{\mathbf{u}_{s i}, \mathbf{N}_{s i}, \mathbf{M}_{s i}\right\} \in \mathscr{D}_{i}$ where $i \in$ $[1, \ldots, n]$ is the index related to the subdomain $\Omega_{i}$ such that

$$
\begin{aligned}
& \sum_{i=1}^{n}\left\{\int_{\partial_{\overline{\mathbf{v}}_{i}} \Omega_{i}} \delta \mathbf{b}_{i}^{H}\left(\mathbf{v}_{s i}-\overline{\mathbf{v}}_{i}\right) \mathrm{d} \mathbf{s}-\int_{\partial_{\overline{\mathbf{b}}_{i}} \Omega_{i}} \delta \mathbf{v}_{i}^{H}\left(\mathbf{b}_{s i}-\overline{\mathbf{b}}_{i}\right) \mathrm{d} \mathbf{s}\right. \\
& +\int_{\partial_{\bar{w}_{i}} \Omega_{i}} \delta q_{i}^{H}\left(w_{s i}-\bar{w}_{i}\right) \mathrm{d} \mathbf{s}-\int_{\partial_{\bar{q}_{i}} \Omega_{i}} \delta w_{i}^{H}\left(q_{s i}-\bar{q}_{i}\right) \mathrm{d} \mathbf{s}
\end{aligned}
$$

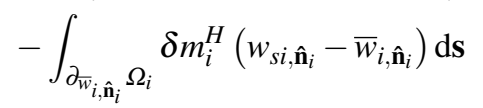

$$
\begin{aligned}
& +\int_{\partial_{\bar{m}_{i}} \Omega_{i}} \delta w_{i, \hat{\mathbf{n}}_{i}}^{H}\left(m_{s i}-\bar{m}_{i}\right) \mathrm{d} \mathbf{s} \\
& +\left.\delta w_{\mathscr{C} i}^{H}\left(q_{\mathscr{C} s i}-\bar{q}_{\mathscr{C} i}\right)\right|_{\partial \partial_{\bar{q}_{\mathscr{C} i}} \Omega_{i}} \\
& -\left.\delta q_{\mathscr{C} i}^{H}\left(w_{\mathscr{C} s i}-\bar{w}_{\mathscr{C} i}\right)\right|_{\partial \partial_{\bar{w}_{\mathscr{C}} i} \Omega_{i}} \\
& +\frac{n_{\Gamma}}{n_{\Gamma}+1} \int_{\Gamma} \delta \mathbf{b}_{i}^{H} \mathbf{v}_{s i}+\delta q_{i}^{H} w_{s i}-\delta m_{i}^{H} w_{s i, \hat{\mathbf{n}}_{i}} \mathrm{~d} \mathbf{s} \\
& -\frac{1}{n_{\Gamma}+1} \int_{\Gamma} \delta \mathbf{v}_{i}^{H} \mathbf{b}_{s i}+\delta w_{i}^{H} q_{s i}-\delta w_{i, \hat{\mathbf{n}}_{i}}^{H} m_{s i} \mathrm{~d} \mathbf{s} \\
& -\left.\frac{n_{\mathscr{C}}}{n_{\mathscr{C}}+1} \delta q_{\mathscr{C} i}^{H} w_{\mathscr{C} s i}\right|_{\mathscr{C}}+\left.\frac{1}{n_{\mathscr{C}}+1} \delta w_{\mathscr{C} i}^{H} q_{\mathscr{C} s i}\right|_{\mathscr{C}} \\
& -\sum_{j=1, j \neq i}^{n}\left\{\frac{1}{n_{\Gamma}+1} \int_{\Gamma} \delta \mathbf{b}_{i}^{H} \mathbf{v}_{s j}+\delta q_{i}^{H} w_{s j}+\delta m_{i}^{H} w_{s j, \hat{\mathbf{n}}_{j}} \mathrm{~d} \mathbf{s}\right. \\
& +\frac{1}{n_{\Gamma}+1} \int_{\Gamma} \delta \mathbf{v}_{i}^{H} \mathbf{b}_{s j}+\delta w_{i}^{H} q_{s j}+\delta w_{i, \hat{\mathbf{n}}_{i}}^{H} m_{s j} \mathrm{~d} \mathbf{s} \\
& \left.\left.-\left.\frac{1}{n_{\mathscr{C}}+1}\left(\delta q_{\mathscr{C} i}^{H} w_{\mathscr{C} s j}+\delta w_{\mathscr{C} i}^{H} q_{\mathscr{C} s j}\right)\right|_{\mathscr{C}}\right\}\right\}=0 \\
& \forall\left\{\delta \mathbf{u}_{i}, \delta \mathbf{N}_{i}, \delta \mathbf{M}_{i}\right\} \in \mathscr{D}_{0 i}
\end{aligned}
$$

where $\mathscr{D}_{s d 0 i}$ is the test function space being VTCR a Galerkin method. Ladevèze in [25] proved uniqueness and existence properties in general elastic theory. Since shell theory is a particularization of such theory, those demonstrations (properly adapted to meet shell theory approximations) hold in our specific case.

\subsection{Shape functions}

Since VTCR is a Trefftz method, any kind of shape function $\mathbf{f}_{S F i}$, proved that satisfy equilibrium equations, can be chosen as solution in subdomain $\Omega_{i}$. Without loss of generality, such family of shape functions is

$$
\mathbf{f}_{S F}\left(\mathbf{x}_{r e l i}\right)=\int_{0}^{2 \pi} a_{i}(\zeta) \hat{\mathbf{c}}_{i}(\zeta) e^{j \mathbf{k}_{i}(\zeta) \mathbf{x}_{r e l i} \mathrm{~d} \zeta}
$$

These functions are "Herglotz wave functions". [41] explains in detail how these functions satisfy the Helmholtz equations. The proof for thin shell equations is alike. For the sake of simplicity, it is omitted. However, it is demonstrated for their discretized version in Section 3.1.4 At this stage no discretizations have been performed and Equation 37 is the exact solution. Wave vector $\mathbf{k}_{i}(\zeta)$ and unit direction vector $\hat{\mathbf{c}}_{i}(\zeta)$ are chosen so that equilibrium equations are identically satisfied for every possible amplitude function $a_{i}(\zeta)$. This is further explained in Section 3.1.4 $\mathbf{x}_{\text {reli }}$ is the relative position vector in curvilinear coordinates $\left\{\alpha_{i}, \beta_{i}\right\}$. Without loss of generality, it is relative to subdomain geometric center. Yet, any other subdomain point can be chosen. $a_{i}(\zeta)$ is an amplitude function and it is set by the weak variational formulation to address boundary and corner conditions. In order to practically calculate integrals of the weak form, this function is discretized.

\subsubsection{Discretizations}

A classical way chosen by Riou in [32] uses a sum of Dirac delta $\delta$

$a_{i}(\zeta) \approx \sum_{l=1}^{n} a_{l i} \delta\left(\zeta_{l}\right)$

that simplified Equation 37 into

$\mathbf{f}_{S F}\left(\mathbf{x}_{r e l i}\right) \approx \sum_{l=1}^{n} a_{l i} \hat{\mathbf{c}}_{l i} e^{j \mathbf{k}_{l i} \mathbf{x}_{r e l}}$,

where $a_{q i}$ are amplitude coefficients determined by the weak form. Since the integral over the unit circle in Equation 39 disappears, other terms $\hat{\mathbf{c}}_{l i}$ and $\mathbf{k}_{l i}$ are discretized too. This drastically reduces complexity of integrals of the weak form. In other words, Riou approximated the solution as a sum of plane waves. The main advantage of this approach is that, in case of "straight boundaries' 2 analytic integrals are possible. This is a dramatic spare of computational time.

Different discretizations such as wave band [32] and Fourier [21] exist in literature and have different advantages (i.e. an inexpensive way to compute a convergence indicator). Yet, the advantage presented before prompted us to chose and improve the plane wave discretization.

As anticipated, wave vectors $\mathbf{k}_{i}(l)$ and unit direction vectors $\hat{\mathbf{c}}_{i}(l)$ of the plane wave are chosen so that equilibrium equations are identically satisfied. Since Dirac delta erases the integral over the unit circle, they are discretized too. Without loss of generality, a discretized wave vector can be decomposed as

$\mathbf{k}_{i}(l)=\mathbf{k}_{l i}=k_{l i} \hat{\mathbf{k}}_{l i}$,

${ }^{2}$ Straight boundaries in the general case of curvilinear coordinates; i.e. in a cylindrical coordinate system a "straight boundary" along a curvilinear coordinate is circular. 
where $\hat{\mathbf{k}}_{l i}$ is a unit direction vector of the wave vector and $k_{l i}$ is the wavenumber. The unit direction vector $\hat{\mathbf{k}}_{l i}$ is set " $\mathrm{a}$ priori" and is part of the discretization process. It is proved in [32] that two plane waves types are needed: propagatives and evanescents. The mathematical difference between them lies in the unit direction vector $\hat{\mathbf{k}}_{l i}$ chosen. In case of propagative waves it is

$\hat{\mathbf{k}}_{l i}=\mathbf{T}_{l i} \cdot\left[\begin{array}{l}1 \\ 0\end{array}\right]$

where $\mathbf{T}_{l i}$

$\mathbf{T}_{l i}=\left[\begin{array}{cc}\cos \left(\theta_{l i}\right) & -\sin \left(\theta_{l i}\right) \\ \sin \left(\theta_{l i}\right) & \cos \left(\theta_{l i}\right)\end{array}\right]$

is a rotation matrix and $\theta_{i} \in[0 ; 2 \pi)$ is a discretized angle of the plane wave direction. Their qualitative behavior is pictured in Figure 5

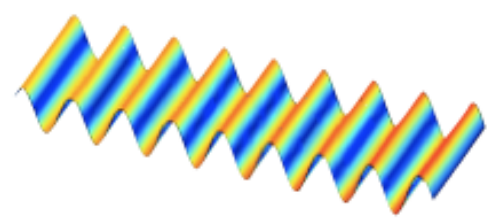

Fig. 5: Qualitative behavior of a propagative wave described in Section 3.1

For the other wave type, in order to enforce evanescent behavior, it is suggested in [32] to use the following formulation

$\hat{\mathbf{k}}_{m i}=\mathbf{T}_{m i} \cdot\left[\begin{array}{c}\sqrt{1+\cos ^{2}\left(\phi_{m i}\right)} \\ j \cos \left(\phi_{m i}\right)\end{array}\right]$

where $\phi_{m i} \in[-\pi ; \pi]$ is a different discretized angle in the complex domain. This angle controls ratio between oscillatory and evanescent parts of the wave. In this case it is

$r_{e o}=\frac{\cos \left(\phi_{m i}\right)}{\sqrt{1+\cos ^{2}\left(\phi_{m i}\right)}}$.

By definition $r_{e o} \in\left[-\frac{1}{2} ; \frac{1}{2}\right]$, therefore, if faster-oscillating evanescent waves exists, they are omitted. In fact, there is no a priori consideration that allows such restriction. For this reason, we modified the formula

$\hat{\mathbf{k}}_{m l i}=\mathbf{T}_{l i} \cdot\left[\begin{array}{c}\cosh \left(\phi_{m i}\right) \\ j \sinh \left(\phi_{m i}\right)\end{array}\right]$
In this case the ratio is

$r_{e o}=\frac{\sinh \left(\phi_{m i}\right)}{\cosh \left(\phi_{m i}\right)}$

This new version has no ratio limits while keeping $\hat{\mathbf{k}}_{m l i}\left(\theta_{l i}, \phi_{m i}\right)$ a unit vector. Due to evanescent behavior, these waves are crucial only along specific boundaries. For this reason, for evanescent waves $l \in\left[1, \ldots, n_{b i}\right]$ where $n_{b i}$ is the number of boundaries of subdomain $\Omega_{i}$. This drastically reduces the number of DoFs of VTCR. Their qualitative behavior is illustrated in Figure 6

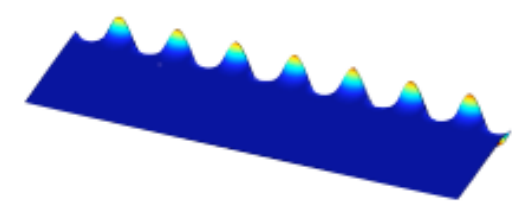

Fig. 6: Qualitative behavior of an evanescent wave described in Section 3.1.

The next Section discusses how to discretize angles $\left\{\theta_{l i}, \phi_{m i}\right\}$ to reduce computational costs.

\subsubsection{Quasi-symmetric ray distribution}

The angle $\theta_{l i} \in[0,2 \pi)$ controls direction of propagative waves. The parameter $\phi_{m i}$ governs ratio $r_{e o}$. Since it is discretized, $\phi_{m i}$ is distributed over $\left[-\phi^{\prime}, \phi^{\prime}\right]$ where $\phi^{\prime}$ is set "a priori" and can be always changed. For the sake of simplicity the range is kept symmetric. In previous works [32, 23, 33] these parameters were distributed symmetrically. This choice imposes a complete matrix recomputation as the number of rays change. For example, let us suppose we computed a VTCR solution of a vibrational problem with three propagative rays and we found it unsatisfactory. We want to add one or two rays. In order to keep a symmetric ray distribution, previous rays must move as illustrated in Figure 7. For this reason previous matrices cannot be reused and must be recomputed anew as ray number change. Since for VTCR matrix creation can be relevant in terms of computational time, this effect can be expensive in terms of computational costs.

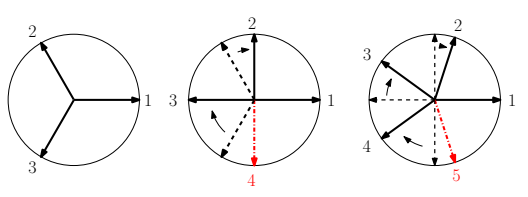

Fig. 7: Effect of symmetric distribution policy during discretization refinement example explained in Section 3.1.2 
Conversely, in the present approach a quasi-symmetric ray distribution is used. In this algorithm previous rays are fixed as new ones are added. The first ray can be placed in any direction. After that, new rays are inserted in gaps among previous rays in the most possible symmetric way. Figure 8 illustrates the algorithm for the first eight rays.

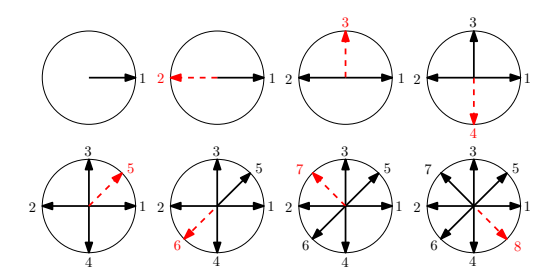

Fig. 8: Quasi-symmetric ray distribution for the first eight rays as described in Section 3.1.2

This method permits complete recycling of old matrices when more rays are added. The drawback is that for a given ray number its distribution could be asymmetric. For this reason, this algorithm could require more rays to reach convergence than a symmetric distribution method. Let us compare a quasi-symmetric and a symmetric distribution of $n$ rays. The shift angle $\theta_{s i}$ of ray $i$ in the quasi-symmetric distribution with respect of the symmetric one is

$\theta_{s i}=\gamma_{i} \frac{2 \pi}{n}$

where $\gamma_{i} \in[0,1]$ is a discrete parameter. Since it varies with $\frac{1}{n}$, the difference between algorithms decreases as the ray number $n$ increases. In practice, when convergence is reached the difference is already negligible. Moreover, in this case

$$
\left\{\begin{array}{c}
\mathscr{F}_{n} \in \mathscr{F}_{m}, \\
n \leq m,
\end{array}\right.
$$

where $\mathscr{F}_{n}$ and $\mathscr{F}_{m}$ are shape function spaces with $n$ and $m$ shape functions respectively. Therefore, except for pollution and resonance effects, an error indicator is a neverincreasing function with the number of shape functions.

For the sake of clarity the present discussion was implicitly developed considering a propagative wave distribution. Yet, it can be extended to evanescent wave distribution.

\subsubsection{Orthotropic materials and generalization to non-cartesian coordinate systems}

Equation 41 and Equation 45 are defined for isotropic materials in a cartesian coordinate system. In this Section corrections for orthotropic materials and non-cartesian coordinate systems are illustrated. Kovalevsky in [23] suggested to add a correction matrix $\mathbf{O}_{i}$ to address orthotropic materials in plates

$\mathbf{k}_{l i}=\mathbf{O}_{i} \hat{\mathbf{k}}_{l i} k_{l i}$,

where

$$
\mathbf{O}_{i}=\sqrt[4]{\rho_{i} h_{i} \omega^{2}}\left[\begin{array}{cc}
D_{x i}^{-1 / 4} & 0 \\
0 & D_{y i}^{-1 / 4}
\end{array}\right]
$$

In this formulation $\mathbf{O}_{i}=\mathbf{O}_{i}\left(\rho_{i} h_{i} \omega^{2}\right)$. This dependency can lead to numerical difficulties during calculation of $k_{l i}$ and $\hat{\mathbf{c}}_{l i}$ because $\mathbf{O}_{i} \hat{\mathbf{k}}_{l i}$ is no more a unit vector. In the present approach we use a slight different formulation that solves such problem and expands its applicability to curvilinear coordinates

$$
\begin{aligned}
\mathbf{k}_{l i} & =\mathbf{L}_{i} \mathbf{O}_{i} \hat{\mathbf{k}}_{l i} k_{l i} \\
\mathbf{O}_{i} & =\sqrt[8]{D_{\alpha i} D_{\beta i}}\left[\begin{array}{cc}
D_{\alpha i}^{-1 / 4} & 0 \\
0 & D_{\beta i}^{-1 / 4}
\end{array}\right] \\
\mathbf{L}_{i} & =\left[\begin{array}{cc}
L_{\alpha i} & 0 \\
0 & L_{\beta i}
\end{array}\right] .
\end{aligned}
$$

In this case coefficients are dimensionless and Lamé parameters are added to address non-cartesian coordinate systems. Such modifications generalize the formulation to curvilinear coordinates while increasing precision in computation of $k_{l i}$ and $\hat{\mathbf{c}}_{l i}$. More details about determination of these two parameters are illustrated in the following Section.

\subsubsection{Wavenumber and unit direction vector of the plane wave determination}

As anticipated, these parameters are chosen so that equilibrium equations are identically satisfied. Substituting a generic shape function defined in Equation 39 with a given unit direction vector $\hat{\mathbf{k}}_{l i}$ in Equation 9 and Equation 10 leads to a linear set of equilibrium equations that in matrix form is

$\mathbf{Z}_{l i} \hat{\mathbf{c}}_{l i} a_{l i}=0$,

where $\mathbf{Z}_{l i}=\mathbf{Z}_{l i}\left(k_{l i}\right)$ is a $3 \times 3$ matrix that depends on $k_{l i}$. In order to obtain untrivial results $\left(a_{l i}=0\right)$ the dispersion equation must be imposed

$\operatorname{det}\left[\mathbf{Z}_{l i}\right]=0$

It provides values of $k_{l i}$ for the specified unit direction vector $\hat{\mathbf{k}}_{l i}$. When $k_{l i}$ is extracted from Equation 55 , the term 
$\hat{\mathbf{c}}_{l i}$ can be obtained by re-injecting $k_{l i}$ in Equation 55 and enforcing that

$\left\|\hat{\mathbf{c}}_{i i}\right\|=1$.

Since $\mathbf{Z}_{l i}$ determinant is set equal to zero and $\hat{\mathbf{c}}_{l i} \neq \mathbf{O}$ is a kernel vector of $\mathbf{Z}_{l i}$, the equilibrium equations are satisfied for every possible amplitude $a_{i}$. These variables are set by a weak form to address boundary, corner, and coupling conditions.

In [32] in-plane inertia is neglected in Equation 55] This simplification leads to a fourth-degree dispersion equation that has four solutions. Half of them can be discarded since concern regressive waves already addressed by the opposite unit direction vector $\hat{\mathbf{k}}_{l^{\prime} i}=-\hat{\mathbf{k}}_{l i}$. The two remaining solutions are a propagative and an evanescent out-of-plane waves. Such simplification drastically reduces number of DoFs (thus computational time) but it is prohibited when out-of-plane behavior is not predominant. In this approach we retain in-plane inertia. For this reason, the dispersion equation is an eighth-degree equation that has eight solutions. As previously, half of them can be discarded. Two of the remaining are out-of-plane waves (one propagative and one evanescent) while the other two are propagative in-plane waves that carry in-plane shear (SS) and normal stresses (NS). In Section 4.2 we demonstrate that these waves are crucial when out-of-plane behavior is not prevalent.

\section{Numerical examples: supported half cylinder}

This Section investigates vibrational responses of a supported half cylinder. First, in-plane inertia effects are studied. For the sake of clarity in this case the material is isotropic. A triple comparison is performed among VTCR with and without in-plane inertia and a FEM reference. After, VTCR with in-plane inertia performances and FEM are confronted over a frequency band. Finally, VTCR corrections for orthotropic materials are investigated. The geometry of the previous example is considered changing the material. Its vibrational behavior is compared for a given frequency with a FEM reference.

\subsection{General description of the vibrational problems}

A complex frame structure is illustrated in Figure 9. Three sub-domains are connected by the same edge. The first two are cylinder parts while the last one is a plate. All boundaries are clamped but left edge where an out-of-plane oscillatory distributed load $\mathbf{p}=[1,0,0]^{\prime} e^{j \omega t} \mathrm{~N} / \mathrm{m}$ is applied. For the sake of simplicity thicknesses are constant $h_{1}=h_{2}=h_{3}=3 \mathrm{~mm}$.



Fig. 9: Boundary and geometric dimensions of the frame structure described in Section 4.1.

\subsubsection{Error indicators}

The focus is on displacement field since it unequivocally defines the solution. In order to summarize results in one relevant visual comparison, displacement magnitude portraits are investigated. In the following Sections two comparison types are performed:

- a VTCR solution with a FEM reference,

- two different VTCR solutions.

The first one is a cross-method confrontation while the second one is not. At mid-frequency a small difference in theories can lead to slightly different frequency responses. Since, at this frequency range there are many wave lengths per subdomain, a small difference in wavenumbers can lead to different displacement magnitude portraits that have almost the same energy. For this reason, we define two different error indicators for each comparison type. Both are based on the kinetic energy

$E_{K}(\mathbf{u})=\frac{1}{2} \rho h \omega^{2} \int_{S} \mathbf{u}^{H} \cdot \mathbf{u d} \mathbf{s}$.

where $S$ denotes surfaces. Direct comparisons of VTCR and FEM displacement magnitude portraits could be non-optimal due to small theory differences that can lead to different solutions at mid-frequency. Therefore, in this case, an error indicator based on a comparison between total energies is used

$\operatorname{err}_{F E M}=\frac{\left|E_{K}\left(\mathbf{u}_{F E M}\right)-E_{K}\left(\mathbf{u}_{V T C R}\right)\right|}{E_{K}\left(\mathbf{u}_{F E M}\right)}$,

where $\mathbf{u}_{F E M}$ and $\mathbf{u}_{V T C R}$ are displacement fields of FEM and VTCR respectively and $|\square|$ denotes the absolute value.

In the following Sections comparisons between VTCR solutions are performed to analyze VTCR convergence. In this case the two solutions share the same theory, thus a more strict error indicator can be considered. It is based on the energy of the displacement magnitude portrait difference

$e r r_{r e l}=\frac{E_{K}\left(\mathbf{u}_{V T C R m}-\mathbf{u}_{V T C R n}\right)}{E_{K}\left(\mathbf{u}_{V T C R m}\right)}$,

where $\mathbf{u}_{V T C R} n$ and $\mathbf{u}_{V T C R} m$ are two different VTCR solutions, $n$ and $m$ denote ray numbers, and $m>n$. 


\subsubsection{Software and convergence criterions}

In the following Sections, VTCR solutions are compared with ABAQUS ${ }^{\circledR}$ tests considered FEM references. In lowfrequency range the rule-of-thumb to set mesh size is

$h_{0}=\frac{\lambda}{10}$,

where $h_{0}$ is the maximum edge length of a mesh element and $\lambda$ is the typical phenomenon wavelength. Equation 60 can be rearranged in terms of wavenumber $k$

$h_{0} k=\frac{2 \pi}{10}$.

Ihlenburg in acoustic [20] and Deraemaeker, Babuška, and Bouillard for general Helmholtz problems [9] proved that this relation is not valid at mid-frequency due to highscattering behavior. In particular, they affirmed that pollution error becomes predominant as the wavenumber increases. They suggested a corrected version of the rule-of-thumb

$h_{0}^{2} k^{3}=\frac{4 \pi^{2}}{100 L}$

where $L$ is a characteristic dimension of the problem considered. Since in shell theory many wave types are present, the following rule-of-thumb is used:

$h_{0}^{2} k_{M A X}^{3}=\frac{4 \pi^{2}}{100 L}$

where $k_{M A X}$ is the greatest wavenumber. By definition, in shells in-plane stiffness is much greater than out-of-plane stiffness; thus $k_{M A X}$ is always the wavenumber of the propagative out-of-plane wave along the direction relative to the highest Lamé parameter.

VTCR is implemented in MATLAB ${ }^{\circledR}$. In the following tests VTCR convergence is studied using Equation 59 where $\mathbf{u}_{V T C R m}$ is a reference solution. Since there are three main ray types, convergence seeking is a three-step process. First, out-of-plane propagative rays are increased until convergence keeping other rays equal to reference. Second, outof-plane evanescent ray convergence is analyzed fixing the number of propagative out-of-plane waves equal to its convergence value determined in the first step. Third, propagative in-plane wave convergence is investigated keeping the number of out-of-plane waves (propagative and evanescent) equal to their respective convergence values. Since out-ofplane waves are the fastest oscillating ones, these rays are the most scattering and should be considered first. Anyhow, every other sequence is possible. Converged rays are used in second and third step because interactions between rays can change convergence error. As side effect, this yields plateaus in convergence graphics of second and third step at the level of the error threshold chosen. This process is cheap in terms of computational time because of matrix recycling enabled by the quasi-symmetric ray distribution algorithm. In fact, once matrices are computed for the reference solution they are reused to analyze convergence.

\subsubsection{Hardware}

All tests (VTCR and FEM) are performed on the same workstation. Its characteristics are reported in Table 1.

\begin{tabular}{lcc}
\hline Cores & 16 & \\
Clock Frequency & 2.4 & $\mathrm{GHz}$ \\
RAM & 50 & $\mathrm{~Gb}$ \\
\hline
\end{tabular}

Table 1: Characteristics of the workstation used for numerical examples described in section 4

\subsection{In-plane inertia analysis}

In this Section the focus is on in-plane inertia effects. A triple comparison among VTCR with and without in-plane inertia and a FEM reference is performed at fixed frequency. Geometry description and boundary conditions of the numerical example are reported in Section 4.1. For the sake of clarity, in this case the material is isotropic. Studied frequency and material properties are reported in Table 2.

\begin{tabular}{lcrl}
\hline$f$ & frequency & 2000 & $\mathrm{~Hz}$ \\
$E$ & Young modulus & 200 & $\mathrm{GPa}$ \\
$v$ & Poisson's ratio & 0.3 & \\
$\rho$ & density & 7800 & $\mathrm{~kg} / \mathrm{m}^{3}$ \\
$\eta$ & damping factor & 0.01 & \\
\hline
\end{tabular}

Table 2: Quantities of interest of the example in Section 4.1

For the reasons reported in Section 4.1.2, a FEM reference with 11,293,452 DoFs is used accordingly with Equation 63. The three-step VTCR convergence analysis with in-plane inertia is reported in Figure 10 Accordingly with discussion in Section 4.1.2 a VTCR reference with many rays is computed for convergence analysis. Error threshold is $e r r \leq 0.01$ for every step. Ray number and type for VTCR reference and converged VTCR are reported in Table 3.

Since without in-plane inertia there are no in-plane propagative waves, convergence analysis for VTCR without inplane inertia is equal to VTCR with in-plane inertia convergence arrested to second step. For the reasons reported in 




(a) First step: propagative out-of-plane ray convergence.

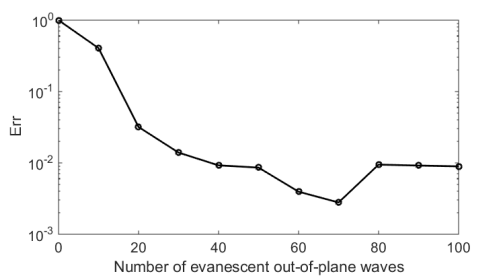

(b) Second step: evanescent out-of-plane ray convergence.



(c) Third step: propagative in-plane ray convergence (normal and shear stress carrying rays).

Fig. 10: three-step convergence process of VTCR with inplane inertia of the numerical example described in Section 4.2 at $2000 \mathrm{~Hz}$. This process is explained in general in Section 4.1.2

\begin{tabular}{ccc}
\hline Types & VTCR reference & converged VTCR \\
\hline Propagative out-of-plane & 200 & 100 \\
Evanescent out-of-plane & 100 & 40 \\
Propagative in-plane (NS) & 100 & 40 \\
Propagative in-plane (SS) & 100 & 40 \\
\hline
\end{tabular}

Table 3: Ray number and type for VTCR reference and converged VTCR with in-plane inertia described in Section 4.2. converged VTCR is the result of the three-step convergence process explained in general in Section 4.1.2 and illustrated for this specific case in Figure 10 where the error threshold is err $\leq 0.01$. NS and SS stands for Normal and Shear Stresses respectively.

Section 4.1.2 the second and the third convergence analysis illustrate a plateau when the error threshold is reached.

The triple comparison among displacement magnitudes of VTCR with and without in-plane inertia and a FEM reference is reported in Figure 11. In this case Equation 58 is used as error indicator due to reasons explained in Section 4.1.1.
VTCR with and without in-plane inertia are confronted with the FEM reference independently. VTCR with in-plane inertia mismatch towards FEM is $\approx 8 \%$ while VTCR without in-plane inertia mismatch towards FEM is above $70 \%$.

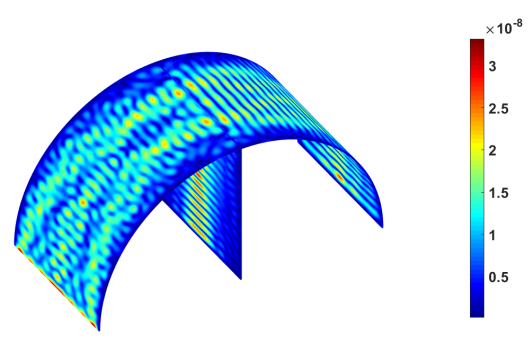

(a) converged VTCR with in-plane inertia.

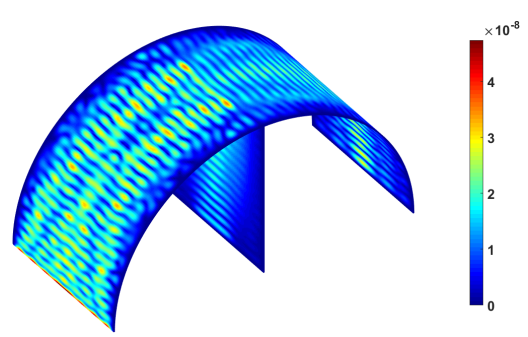

(b) FEM reference.



(c) converged VTCR without in-plane inertia.

Fig. 11: Triple comparison of displacement magnitudes of VTCR with and without in-plane inertia and a FEM reference of the numerical example described in Section 4.2.

Remarks In this numerical example out-of-plane behavior could be considered predominant due to the out-of-plane load and intrinsic shell stiffness difference. Yet, triple comparison illustrated in Figure 11 and error difference confirm necessity of in-plane normal and shear stress carrying rays. Therefore, we can conclude that, for what concerns VTCR, in-plane rays cannot be neglected even if out-of-plane behavior seems predominant.

\subsection{Performance analyzes over a frequency band}

This Section compares VTCR with in-plane inertia and FEM performances over a frequency band. They are divided in 
memory consumption and total computational time. The numerical problem considered is equal to that one illustrated in Section 4.2 but frequency that, in this case, varies over a range $f \in[2000 ; 4000] \mathrm{Hz}$ with a $100 \mathrm{~Hz}$ step. The rule-ofthumb reported in Equation 62 sets the FEM DoFs number. VTCR convergence is analyzed using the three-step process described in Section 4.1.2. For the sake of simplicity convergence is investigated only at extremes $(2000 \mathrm{~Hz}$ convergence analysis is reported in Section 4.2. The general convergence analysis procedure is illustrated in Section 4.1.2. Error threshold is err $\leq 0.01$. For other frequencies ray numbers are linearly interpolated between converged ray numbers reported in Table 3 and Table 4 . This is a conservative choice if $n_{r \beta} \propto f^{\alpha}$ where $n_{r \beta}$ is the ray number of type $\beta$ and $\alpha \geq 1$. This relation is generally satisfied because equilibrium equations are $\propto \omega^{2}$. A more rigorous study of the relation between frequency and converged ray numbers is indeed an interesting topic. Nevertheless, it is out of the aims of this work.

The three-step convergence analysis at $4000 \mathrm{~Hz}$ is illustrated in Figure 12. Converged ray numbers and reference ray numbers are reported in Table 4.

\begin{tabular}{ccc}
\hline Types & VTCR reference & converged VTCR \\
\hline Propagative out-of-plane & 200 & 130 \\
Evanescent out-of-plane & 100 & 40 \\
Propagative in-plane (NS) & 100 & 40 \\
Propagative in-plane (SS) & 100 & 40 \\
\hline
\end{tabular}

Table 4: Ray number and type for VTCR reference and converged VTCR described in Section 4.3 at $4000 \mathrm{~Hz}$. converged VTCR is the result of the three-step convergence process explained in general in Section 4.1.2 and illustrated for this specific case in Figure 12 where the error threshold is err $\leq 0.01$.

Performance analyses over the frequency band [2000;4000] $\mathrm{Hz}$ are reported in Figure 13b and Figure 13c. DoFs cannot be directly compared because methods are intrinsically different. Nevertheless, for the sake of completeness, DoFs required over the frequency band are illustrated in Figure 13a.

Remarks At mid-frequency FEM suffers of pollution effect. DoFs number and time and memory consumptions become prohibitive. Conversely, VTCR is unaffected as supported by Figure 13. Since VTCR remains stable, computational cost differences grow as frequency increases. Figure 13b and Figure 13c illustrate that time and memory consumption differences are already of some orders of magnitude and increase with frequency. Due to differences in memory and computational costs, VTCR could have been run on much less powerful machine (i.e. a laptop) than FEM (which needs a workstation). Therefore, we can affirm that VTCR



(a) First step: propagative out-of-plane ray convergence.

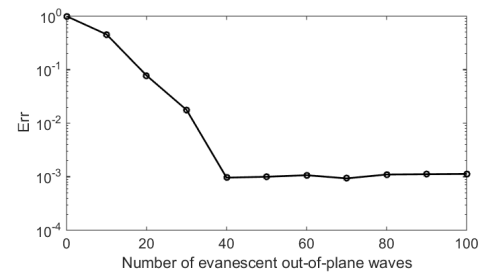

(b) Second step: evanescent out-of-plane ray convergence.

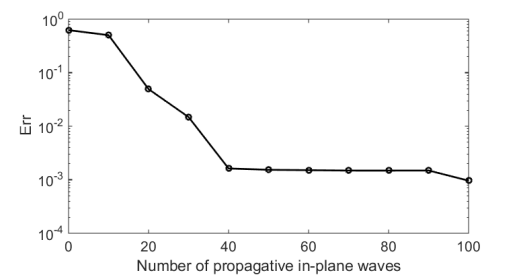

(c) Third step: propagative in-plane ray convergence (normal and shear stress carrying rays).

Fig. 12: three-step convergence process of VTCR with inplane inertia of the numerical example described in Section 4.3 at $4000 \mathrm{~Hz}$. This process is explained in general in Section 4.1.2.

greatly outperform FEM at mid-frequency in terms of computational time and memory consumption. 




(a) DoFs.



(b) Time consumption.



(c) Memory consumption

Fig. 13: Performance analyzes over a frequency band [2000; 4000] Hz of the numerical example described in Section 4.3. VTCR and FEM are compared considering time and memory consumptions. DoFs required are reported for the sake of completeness, a direct comparison is unmeaningful due to theory differences.

\subsection{Orthotropic materials}

This Section tests VTCR corrections for orthotropic materials explained in Section 3.1.3 A VTCR with in-plane inertia solution is compared with a FEM reference at fixed frequency. The numerical example investigated is the supported half cylinder described in Section 4.1. In this case the material is a typical aerospace composite. Table 5 reports its characteristics as well as frequency studied.

\begin{tabular}{ccrl}
\hline$f$ & frequency & 3700 & $\mathrm{~Hz}$ \\
$E_{\theta 1}=E_{\theta 2}=E_{z 3}$ & Young moduli & 125 & $\mathrm{GPa}$ \\
$E_{y 1}=E_{y 2}=E_{y 3}$ & Young moduli & 60 & $\mathrm{GPa}$ \\
$G_{\theta y 1}=G_{\theta y 2}=G_{z y 3}$ & Shear moduli & 18 & $\mathrm{GPa}$ \\
$v_{\theta y 1}=v_{\theta y 2}=v_{z y 3}$ & Poisson's ratios & 0.3 & \\
$\rho_{1}=\rho_{2}=\rho_{3}$ & densities & 2000 & $\mathrm{~kg} / \mathrm{m}^{3}$ \\
$\eta_{\theta 1}=\eta_{\theta 2}=\eta_{z 3}$ & damping factors & 0.001 & \\
$\eta_{y 1}=\eta_{y 2}=\eta_{y 3}$ & damping factors & 0.001 & \\
$\eta_{G 1}=\eta_{G 2}=\eta_{G 3}$ & damping factors & 0.001 & \\
\hline
\end{tabular}

Table 5: Orthotropic material properties and frequency examined of the numerical example described in Section 4.4.
A FEM reference solution is calculated using the ruleof-thumb reported in Equation 63 At this frequency 34,547,616 DoFs are needed to reach convergence due to pollution. Section 4.1.2 further discusses this effect. VTCR convergence is studied by a three-step process described in Section 4.1.2 It is reported in Figure 14 Table 6 illustrates VTCR reference and converged ray numbers summarizing convergence analysis results. Section 4.1.1 discusses errors in detail. For VTCR convergence Equation 59 is used. It is considered acceptable if $e r r \leq 0.01$.

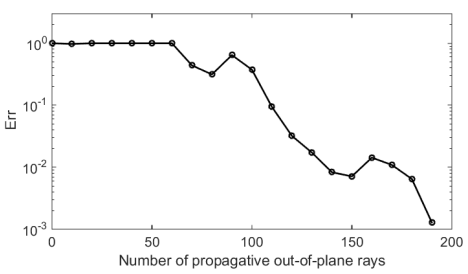

(a) First step: propagative out-of-plane ray convergence.



(b) Second step: evanescent out-of-plane ray convergence.

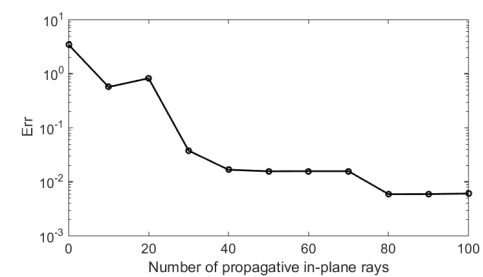

(c) Third step: propagative in-plane ray convergence (normal and shear stress carrying rays).

Fig. 14: three-step convergence process of VTCR with inplane inertia of the numerical example described in Section 4.4 at $3700 \mathrm{~Hz}$. This process is explained in general in Section 4.1.2

Displacement magnitude comparison is depicted in Figure 15. Due to reasons discussed in Section 4.1.1 in this case $\operatorname{err}_{F E M}$ is used. Even if VTCR and FEM solutions are not equal the error based on kinetic energy is $\approx 8 \%$.

Remarks The small error confirms that orthotropic materials are correctly addressed and included in VTCR. This improvement allows the study of a great variety of composite 


\begin{tabular}{ccc}
\hline Types & VTCR reference & converged VTCR \\
\hline Propagative out-of-plane & 200 & 140 \\
Evanescent out-of-plane & 100 & 69 \\
Propagative in-plane (NS) & 100 & 40 \\
Propagative in-plane (SS) & 100 & 40 \\
\hline
\end{tabular}

Table 6: Ray number and type for VTCR reference and converged VTCR described in Section 4.3 at $3700 \mathrm{~Hz}$. converged VTCR is the result of the three-step convergence process explained in general in Section 4.1.2 and illustrated for this specific case in Figure 14 where the error threshold is err $\leq 0.01$.

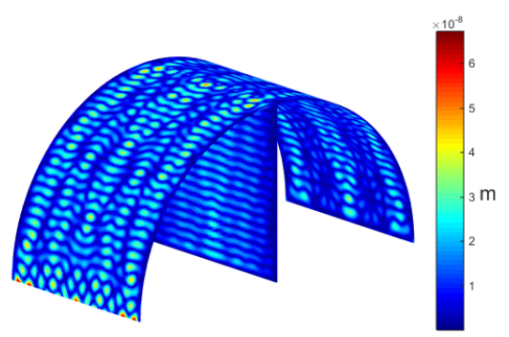

(a) VTCR.



(b) FEM.

Fig. 15: Comparison of displacement magnitude portraits.

shell structures effectively extending VTCR applicability to common composite aerospace and automotive structures.

\section{Conclusions}

In order to extend VTCR to orthotropic shell structures where in-plane inertia can be relevant, many adjustments were proposed. In-plane inertia was addressed using two types of propagative waves (carrying shear and normal stresses). A quasi-symmetric ray distribution algorithm was proposed to reuse matrices. It dramatically reduces computational time when converged ray numbers are unknown or when boundary conditions are changed. Othotropic materials were introduced and a correction matrix for the wave vector was proposed. Differently from previous works [23], it is dimensionless providing additional robustness to the method. Finally, VTCR theory was generalized for non-cartesian coordinate systems.

A numerical example presented in Section 4.1 was deeply presented. Section 4.2 tested VTCR in-plane propagative waves. For the sake of clarity in this case the material was isotropic. Even if there were just an out-of-plane load, inplane behavior was relevant; in fact VTCR with in-plane propagative rays displayed much better results than VTCR without them. After that, VTCR performances were studied in Section 4.3 confronting computational time and memory consumption with a FEM reference. Results illustrate that VTCR greatly outperforms FEM at mid-frequency. Section 4.4 focused on testing VTCR orthotropic material corrections. The previous numerical example was investigated using a classic aerospace composite material and compared with a FEM reference. The error indicator confirmed that there were good correspondence between VTCR and FEM solutions validating orthotropic corrections.

The future work will couple VTCR with a Reduced Order Model (ROM) method to further improve performances.

\section{References}

1. Babuska I, Melenk JM (1995) The partition of unity finite element method. Tech. rep., DTIC Document

2. Barbarulo A, Ladevèze $\mathrm{P}$, Riou $\mathrm{H}$, Kovalevsky L (2014) Proper Generalized Decomposition applied to linear acoustic: A new tool for broad band calculation. J Sound Vib 333(11):2422-2431, DOI 10.1016/j. jsv.2014.01.014

3. Bouillard P, Suleaub S, Suleau S (1998) Element-Free Galerkin solutions for Helmholtz problems: fomulation and numerical assessment of the pollution effect. Comput Methods Appl Mech Eng 162(1-4):317-335, DOI 10.1016/S0045-7825(97)00350-2

4. Cessenat O, Despres B (1998) Application of an ultra weak variational formulation of elliptic PDEs to the two-dimensional Helmholtz problem. SIAM J Numer Anal 35(1):255-299 
5. Chevreuil M, Ladevèze P, Rouch P (2007) Transient analysis including the low- and the medium-frequency ranges of engineering structures. Comput Struct 85(1718):1431-1444, DOI 10.1016/j.compstruc.2006.08.091

6. De Rosa S, Franco F (2010) On the use of the asymptotic scaled modal analysis for time-harmonic structural analysis and for the prediction of coupling loss factors for similar systems. Mech Syst Signal Process 24(2):455-480, DOI 10.1016/j.ymssp.2009.07.008

7. Deckers E, Van Genechten B, Vandepitte D, Desmet W (2011) Efficient treatment of stress singularities in poroelastic wave based models using special purpose enrichment functions. Comput Struct 89(11):11171130

8. Deckers E, Hörlin NE, Vandepitte D, Desmet W (2012) A Wave Based Method for the efficient solution of the 2D poroelastic Biot equations. Comput Methods Appl Mech Eng 201-204:245-262, DOI 10.1016/j.cma.2011. 09.015

9. Deraemaeker A (1999) Dispersion and pollution of the FEM solution for the Helmholtz equation in one, two and three dimensions. Int J Numer Methods Eng 46(4):471-499, DOI 10.1002/(SICI)1097-0207(19991010)46:4〈471:: AID-NME684>3.0.CO;2-6

10. Desmet W, Van Hal B, Sas P, Vandepitte D, Hal BV (2002) A computationally efficient prediction technique for the steady-state dynamic analysis of coupled vibroacoustic systems. Adv Eng Softw 33(7):527-540, DOI 10.1016/S0965-9978(02)00062-5

11. Farhat C, Roux FX (1991) A method of finite element tearing and interconnecting and its parallel solution algorithm. Int J Numer Methods Eng 32(6):1205-1227

12. Farhat C, Harari I, Franca LP (2001) The discontinuous enrichment method. Comput Methods Appl Mech Eng 190:6455-6479

13. Franca LP, Frey S, Hughes TJ (1990) Stabilized finite element methods. INRIA

14. Genechten BV, Vandepitte D, Desmet W (2011) A direct hybrid finite element wave based modelling technique for efficient coupled vibro-acoustic analysis. Comput Methods Appl Mech Eng 200(5):742-764, DOI 10.1016/j.cma.2010.09.017

15. Hall WS (1994) Boundary Element Method. Springer

16. Hughes T, Cottrell J, Bazilevs Y (2005) Isogeometric analysis: CAD, finite elements, NURBS, exact geometry and mesh refinement. Comput Methods Appl Mech Eng 194(39-41):4135-4195, DOI 10.1016/j.cma.2004. 10.008

17. Hughes TJR (1995) Multiscale phenomena: Green's functions, the Dirichlet-to-Neumann formulation, subgrid scale models, bubbles and the origins of stabilized methods. Comput Methods Appl Mech Eng 127(1-
4):387-401

18. Hughes TJR (2012) The finite element method: linear static and dynamic finite element analysis. Courier Dover Publications

19. Huttunen T, Gamallo P, Astley RJ (2006) Comparison of two wave element methods for the Helmholtz problem. Commun Numer Methods Eng 25(1):35-52, DOI 10.1002/cnm.1102

20. Ihlenburg F (1998) Finite element analysis of acoustic scattering. Springer

21. Kovalevsky L, Ladevèze P, Riou H (2012) The Fourier version of the Variational Theory of Complex Rays for medium-frequency acoustics. Comput Methods Appl Mech Eng 228(0):142-153

22. Kovalevsky L, Ladevèze P, Riou H, Bonnet M (2012) The variational theory of complex rays for threedimensional helmholtz problems. J Comput Acoust pp 1-25, DOI 10.1142/S0218396X1250021X

23. Kovalevsky L, Riou H, Ladevèze P (2014) A Trefftz approach for medium-frequency vibrations of orthotropic structures. Comput Struct 143:85-90, DOI 10.1016/j. compstruc.2014.07.011

24. Ladevèze $P$ (1996) A New Computational Approach for Structure Vibrations in the Medium Frequency Range. Comptes Rendus Académie des Sci Paris 332(2b):849856

25. Ladevèze P, Rouch P, Riou H, Bohineust X (2003) Analysis of medium-frequency vibrations in a frequency range. J Comput Acoust 11(2):255-283, DOI 10.1142/S0218396X0300195X

26. Langley RS (1992) A wave intensity technique for the analysis of high frequency vibrations. J Sound Vib 159(3):483-502

27. Liu Y (2009) Fast multipole boundary element method: theory and applications in engineering. Cambridge university press

28. Lyon RH (1975) Statistical energy analysis of dynamical systems: theory and applications. MIT press Cambridge, MA

29. Mace B (2003) Statistical energy analysis, energy distribution models and system modes. J Sound Vib 264(2):391-409, DOI 10.1016/S0022-460X(02) 01201-4

30. Monk P, Wang DQ (1999) A least-squares method for the helmholtz equation. Comput Methods Appl Mech Eng 175(1):121-136

31. Perrey-Debain E, Trevelyan J, Bettess P (2004) Wave boundary elements: a theoretical overview presenting applications in scattering of short waves. Eng Anal Bound Elem 28:131-141

32. Riou H, Ladevèze P, Rouch P (2004) Extension of the variational theory of complex rays to shells for medium-frequency vibrations. J Sound Vib 272(1):341- 
360, DOI 10.1016/S0022-460X(03)00775-2

33. Riou H, Ladeveze P, Sourcis B (2008) The multiscale VTCR approach applied to acoustics problems. J Comput Acoust 16(4):487-505, DOI 10.1142/ S0218396X08003750

34. Simpson RN, Scott Ma, Taus M, Thomas DC, Lian H (2014) Acoustic isogeometric boundary element analysis. Comput Methods Appl Mech Eng 269:265-290, DOI 10.1016/j.cma.2013.10.026

35. Soize C (1998) Reduced models in the medium frequency range for general external structural-acoustics systems. J Acoust Soc Am 103(6):3393-3406, DOI $10.1121 / 1.423052$

36. Strouboulis T, Hidajat R (2006) Partition of unity method for Helmholtz equation: q-convergence for plane-wave and wave-band local bases. Appl Math 51(2):181-204

37. Tezaur R, Kalashnikova I, Farhat C (2014) The discontinuous enrichment method for medium-frequency Helmholtz problems with a spatially variable wavenumber. Comput Methods Appl Mech Eng 268:126-140, DOI 10.1016/j.cma.2013.08.017

38. Van der Heijden AMA, Hutchinson J, Achenbach J (2008) WT Koiter's elastic stability of solids and structures. Cambridge Univ Press Cambridge

39. Ventsel E, Krauthammer T (2001) Thin plates and shells: theory: analysis, and applications. CRC press, Basel

40. Wilson D, Hopkins C (2015) Analysis of bending wave transmission using beam tracing with advanced statistical energy analysis for periodic box-like structures affected by spatial filtering. J Sound Vib 341:138-161, DOI 10.1016/j.jsv.2014.12.029

41. Zhang D, Ma F, Zheng E (2013) A Herglotz wavefunction method for solving the inverse Cauchy problem connected with the Helmholtz equation. J Comput Appl Math 237(1):215-222, DOI 10.1016/j.cam.2012.07.026 